\title{
Load Shedding Frequency Management of Microgrids Using Hierarchical Fuzzy Control
}

\author{
Mehran Keivanimehr, Member, IEEE, Harold R. Chamorro, Senior Member, IEEE, \\ Mehdi Zareian-Jahromi, Member, IEEE Felix Rafael Segundo-Sevilla, Senior Member, IEEE \\ Josep M. Guerrero, Fellow, IEEE, and Charalambos Konstantinou, Senior Member, IEEE
}

\begin{abstract}
Frequency control is one important issue in autonomous operating mode of Microgrids (MGs). In stand-alone microgrids, when generation power is not enough, it is inevitable to shed some parts of the load. This paper presents a decentralized fuzzy controller for optimal load shedding in MGs. The proposed controller which is separately installed for each load center uses a hierarchical fuzzy controller approach to make the optimal decision. Three inputs of the hierarchical fuzzy controller are the power of non-critical load, the load energy not supplied and its interruption frequency. From a specific time of the past, these inputs are calculated for all load centers, and updated periodically. The non-critical part of the loads which can be shed to survive network. Each decentralized controller of the loads has a bilateral communication with the microgrid central controller to transact some information, periodically. Simulation results on a typical microgrid with several load buses showing the effectiveness of the proposed method.
\end{abstract}

Index Terms-Smart Microgrids, Load Frequency Control, Hierarchical Fuzzy Control, Distributed Generation, Load Shedding

\section{INTRODUCTION}

$\mathbf{M}$ Icrogrids are playing an increasingly important role in the evolving electricity environment, which is characterized by growing Distributed Energy Resources (DER) penetration and active customer participation [1], [2]. However, technical challenges have been emerging attracting their research attention [3]. With growing number of these cellular energy systems, the operation and planning of microgrids integration requires to be studied further more [4].

Load Frequency Control (LFC) is one of the challenges in microgrids operation that requires to be investigated. This challenge is more critical when MGs operate in stand-alone mode [5], since of the small scale of MGs, outage of generators or abrupt variations in load demand may result in a considerable frequency deviation. The frequency drop and Rate of Change

Mehran Keivanimehr is with the Department of Electrical Engineering, University of Kashan, Kashan, Iran (mehrankeivanimehr@gmail.com).

Harold R. Chamorro is with KTH, Royal Institute of Technology, Stockholm, Sweden. (hr.chamo@ieee.org).

Mehdi Zareian-Jahromı 1s with School of Electrical and Computer Engineering, Shiraz University, Shiraz, Iran. (m.jahromi@aut.ac.ir).

Felix Rafael Segundo-Sevilla is with Power Systems and Smart Grid Lab at the Zurich University of Applied Sciences, Zurich, Switzerland, (e-mail: segu@zhaw.ch)

Josep M. Guerrero is with Aalborg University, Aalborg, Denmark (email: joz@et.aau.dk)

Charalambos Konstantinou is with the Division of Computer, Electrical and Mathematical Sciences and Engineering, King Abdullah University of Science and Technology (KAUST), Thuwal 23955, Saudi Arabia (ckonstantinou@ieee.org). of Frequency (RoCoF) depend on the both size and type of generation units' outage and loads involved [6]. In stand-alone operating mode, should be available enough generation reserve in MGs to compensate active power mismatch, otherwise a portion of loads should be shed to prevent the black out of MG [7].

Recently, many researches have been done to analyze and improve the frequency control in MGs [8], [9]. In conventional distribution power networks when a shortage of active power generation happens, a number of electrical loads must be shed accordingly based on the load priorities as shown in [10]. The priority of these loads' selection is based on three parameters: the "importance" of electrical loads, the amount of energy not supplied and, the number of interruptions occurs [11]. In non-mechanized system and without any optimization between these parameters, distribution operator determines proper electrical loads that must be disconnect. Consequently, power network reliability indices will get worse over time [12].

Reference [13] proposes a fuzzy programming algorithm for load shedding in order to control the frequency when there is not enough generation capacity in an autonomous MG. Reference [14] proposes a method of controlling frequency and voltage of MGs that use storage devices and load shedding strategy. Also, reference [15] uses a Proportional Integral (PI) controller for frequency control. The load shedding problem in power system has been solved by many mathematical techniques, such as linear programming [16], nonlinear programming [17], and the interior point method [18]. These algorithms are fast, but they use some approximation and some valuable indices such as reliability indices which are not considered [19].

Based on a Decentralized Control System (DCS) architecture, in this paper, a new method is proposed for optimal load shedding for frequency control of microgrids regarding the reliability indices, to have a balance among the non-critical loads in load shedding process. In the proposed method, the integrated load center is controlled by an Intelligent Load Controller (ILC). Thus, a Hierarchical Fuzzy Controller (HFC) is suggested for ILCs in order to control the non-critical part of the loads. In a high dimensional fuzzy system, HFC in compare to the conventional fuzzy controller has a rapid and simple calculation [20]. Based on three inputs, the amount of power of non-critical load, interruption frequency and energy not supplied, the proposed HFC determines a time-dependent shedding curve which is used in ILC. The proposed method is applied to a test system with satisfactory results. 
The rest of this paper is organized as follows: Section II describes the microgrid model and the load shedding challenge. Section III briefly explains the HFC method. Section IV presents the load shedding scheme proposed. Section V presents the results obtained and discusses the simulations obtained under two scenarios. Finally, the conclusion are given in Section in VI

\section{MicROGRID MODELING}

A MG consists of some dispersed generations which are usually based on renewable energies, some load points and a hierarchical control system [21]. One of the most significant specificity of MGs is that the generators and electricity loads are in small geographical zone; this reduces the length of power transmission line and losses in MGs. In these small power systems some problems like: power quality, voltage and frequency control, protection and stability of microgrids should be re-evaluated.

Fig. 11 shows a typical MG with three different micro sources, three load buses and communication link provided for transferring data between MG's components whereas MG can be expanded. The control scheme comprises separate Intelligent Micro source Controllers (IMCs), ILC and MG Central Controller (MGCC). IMCs control Distributed Generators (DGs) with setting the generation set point of each DG. Also, ILC is used in order to control the interruptible loads. The MGCC controls the MG through local IMCs and ILCs and also coordinates the MG with the upstream system and other neighbor MGs through Distribution Management System (DMS).

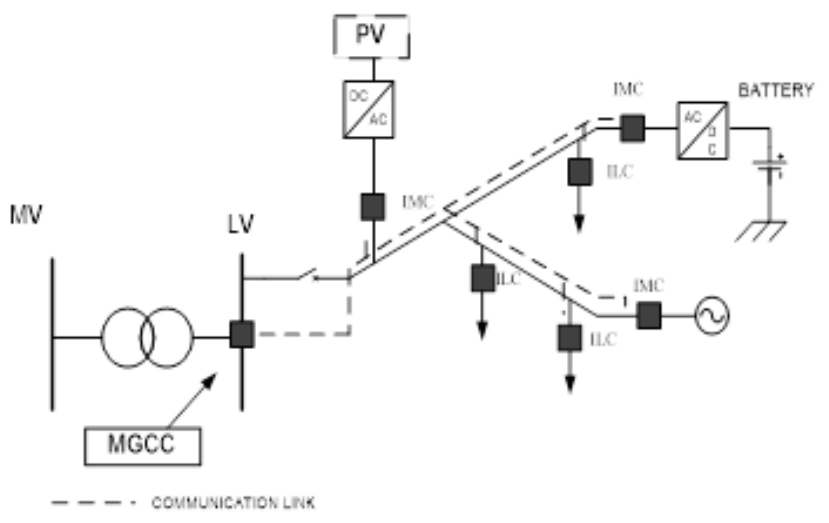

Fig. 1: Microgrid Model

Based on DCS, communication link can provide a platform to transfer data between ILC, IMC and MGCC. The rate of information transact in $\mathrm{MG}$ depends on communication media and protocol. IEC 61850, DNP3, etc. are used as communication protocols for MGs. Recent researches show that the IEC 61850 is a proper communication protocol for MGs that has standardized formats or templates for exchanging data between different device controllers [22].

In the proposed MG model, data of control system exchanges every 0.5 seconds between MGCC, ILC and IMC.

\section{Hierarchical Fuzzy Control Preliminaries}

Standard fuzzy systems have been used for approximation and control systems, widespread and successfully [14], [23]. When a fuzzy system is used in multi-dimensional and complicated systems, the number of fuzzy controller inputs increase and this causes multiple problems; namely, high computational burden and more complicated fuzzy rules. To solve these challenges some methods were proposed [24]. However, amongst these methods Hierarchical Fuzzy System (HFS) is the useful and practical one [25].

The Hierarchical Fuzzy System (HFS) was developed by Raju and Zhou at the beginning of 1990's [26].This method has been used in many applications by experts and researchers [27]. In HFS method instead of high-dimensional standard fuzzy system, some of low-dimensions in hierarchical layers are used. Fig. 2 shows the block diagram of a HFS with several layers. It contains some low-dimension subsystems established consecutively.

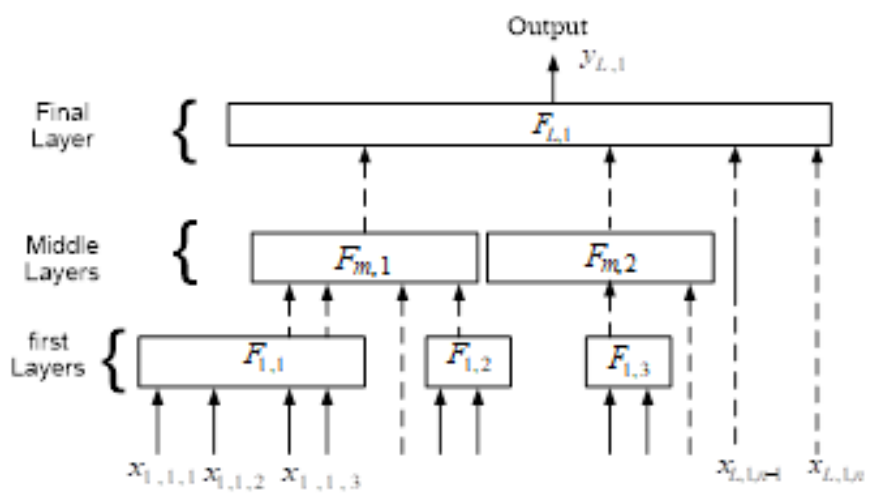

Fig. 2: General Structure of the Hierarchical Fuzzy System

The main point of this structure is the same type inputs arranged together and make a fuzzy subsystem. The best arrangement for HFS is that to put together the matched inputs for each subsystem and use the output of lower subsystem for input of upper subsystem. In complicated and advanced systems, it is difficult to find the proper inputs for subsystem. Wang and his colleagues proposed a hierarchical fuzzy system with hierarchical layers; some subsystems are located in each layer and there is a main input for each subsystem and other inputs are provided from the lower layers. To determine the appropriate structure of hierarchical fuzzy systems and find out dependent variables for analyzing the system, they utilized the Gradient Descent as a criterion. As a role, a fuzzy subsystem (the $p$-th fuzzy subsystem in the $L$-th layer) is represented in the following:

$$
\begin{array}{r}
\hat{y}_{l, p}=f_{l, p}\left(\hat{y}_{l-1, p, 1}, \hat{y}_{l-1, p, 2}, \cdots,\right. \\
\hat{y}_{l-1, p}, p l-1, x_{l-1, p, 1}, \cdots, x_{l-1, p, Q l-1, p}
\end{array}
$$

where $\hat{y}_{l, p}$ is the output of fuzzy subsystem, $f_{l, p}, \hat{y}_{l-1, p, j}$ is the output from fuzzy subsystem, $f_{l-1, p, j}$ (the $j$-th fuzzy subsystem of the (l1)-th layer) to $f_{l-1, P l-1}$ is the total number of outputs from the (l1)-th layer to $f_{l-1, p, j}$, is the $j$-th original 
input variable to $f_{l-1, p, j}$, and $f_{l-1, p}$ is the total number of original input variables to $f_{l, p}[28]$.

In the original form of fuzzy controllers, thus increasing the number of variables, number of rules increases exponentially. Consequently, the calculation time in fuzzy decision making depends on the number of input variables. Equation (2) presents the relationship between variable inputs $(v)$, membership functions $(m)$ and the number of rules $(r)$ :

$$
r=m^{v}
$$

Hence, it is impractical to implement a conventional fuzzy system when the number of input variables increases [18]. In conventional fuzzy system when the inputs of system increase, it is difficult to define the correct and proper rules whereas the rules increase exponentially. In result, to avoiding exponential growth of fuzzy rules it is recommended to use HFS to have a simplified and low error fuzzy system [29].

\section{Proposed LoAd Shedding Scheme}

Frequency control is crucial importance for secure and reliable operation of the MGs system. If the system cannot prevent frequency drop especially in stand-alone operating mode, it may cause blackout in the MG. Therefore, a crucial need for a suitable and fast controller is felt in the MG. It is notable that the main reason of frequency drop in a system is the mismatch between active power generation and demand. Load shedding is a prevalent and useful method to control the system frequency drop. To implement load shedding, each load bus of the system can be divided into two different parts: critical and non-critical parts. The non-critical part can be shed to maintain the frequency of MG in an acceptable range.

In a MG, it is desirable to enhance the system reliability. Therefore, Energy Not Supplied (ENS) and Interruption Frequency (IF) which are two important reliability indices are used in the load shedding process in addition to the Power of Non-Critical (PNC) load. Some researches propose using fuzzy controller for load frequency control in MGs [10]. Based on measurement system and all of ILCs data that exchange every 0.5 seconds between ILCs and MGCC, the inputs of every ILCs must be calculated and normalized, in compare to other loads in MG to make a fair decision. The first input is normalized power of non-critical $\left(\hat{P}_{n c}\right)$ part of every load is defined as according (3) :

$$
\hat{P}_{n c}=\frac{P_{n c}}{P_{\max }}
$$

where the $\hat{P}_{n c}$ is the instantaneous power of non-critical part of load and $P_{\max }$ is the maximum value of $P_{n c}$ of loads. The energy not supplied $E_{n s}(i+1)^{k}$ is calculated according to (4) based on last value of energy not supplied $E_{n s}(i)^{k}$ for the $k$-th load until the last interruption time $\Delta t$ of power of non-critical $\left(P_{n c}\right)$ :

$$
E_{n s}(i+1)^{k}=E_{n s}(i)^{k}+P_{n c}^{k} \Delta t
$$

The normalized value of $E_{n s}$ is calculated according to (5) is the next input of the ILC:

$$
\hat{E}_{n s}(i)^{k}=\frac{E_{n s}(i)^{k}-E_{n s-\min }(i)}{E_{n s-\max }(i)-E_{n s-\min }(i)}
$$

The normalized value of the interruption of frequency is considered (6):

$$
\hat{I F}_{i}=\frac{I F_{i}}{I F_{\max }}
$$

where $I F_{i}$ is number of $i$-th load interruptions; and $I F_{\max }$ is the maximum number of interruptions between non-critical loads. Consequently, to use the proposed indices as variable inputs of the load frequency controller, this paper proposes to employ a Hierarchical Fuzzy Controller (HFC). As an important feature of HFC, these kinds of controllers are very robust and insensitive against variations in the power network [8]. Typical schematic of HFC is shown in Fig. 3. In the proposed algorithm, ENS and IF are inputs of the first fuzzy logic block; PNC and the output of the first block in HFC are determined as inputs of the second block.

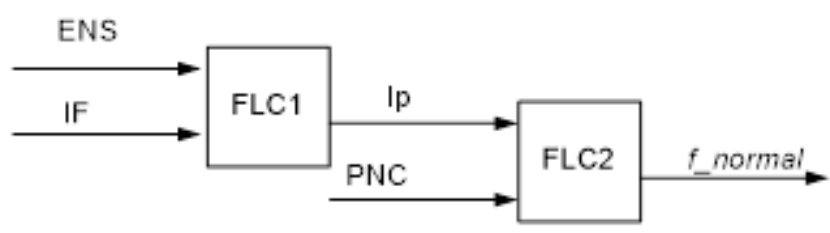

Fig. 3: Hierarchical Fuzzy Controller Schematic

As it is depicted in Fig. 4, the membership functions of the ENS are considered to be triangular functions. Similar to ENS, the membership functions of IF and PNC are triangular. The fuzzy variables are then expressed by linguistic terms such as "Very small(VS)," "Small (S)," "Medium (M)," "Large (L)," "Very Large (VL)," "Smallest (SEST)," and "Smaller (SER)" "Larger (LER)," "Largest (LEST)," for the input and output. Fuzzy rules of Fuzzy Logic Controller (FLC) block are shown in Table I and III

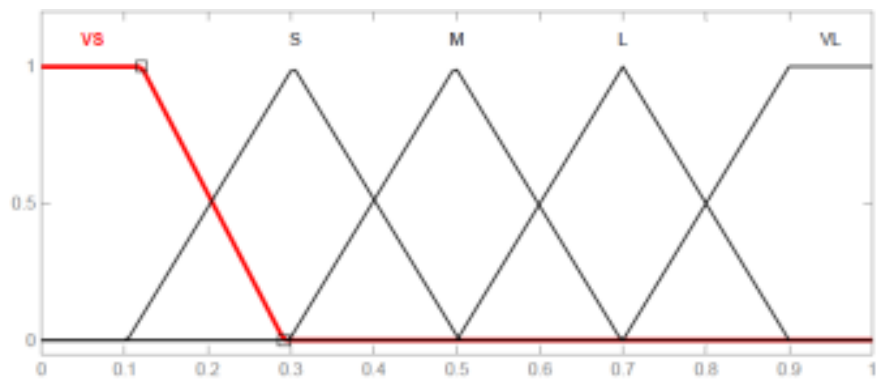

Fig. 4: Membership set for the input variable ENS

According to communication link between MGCC, ILCs and IMCs, the input variables of HFCs are frequently received from MGCC. It is notable that the amounts of input variables are determined by MGCC according to system data. In this paper all input variables are normalized by MGCC to improve 
TABLE I: Fuzzy rule of first FLC

\begin{tabular}{|c|c|c|c|c|c|c|}
\hline & & & & & & \\
\hline \hline & & VS & S & M & L & VL \\
\hline & VS & VL & VL & L & L & L \\
\hline & S & VL & L & L & M & M \\
\hline & M & L & L & M & S & S \\
\hline & L & M & M & S & S & VS \\
\hline & VL & S & S & VS & VS & VS \\
\hline
\end{tabular}

TABLE II: DG and V2G Size and Location

\begin{tabular}{|c|c|c|c|c|c|c|}
\hline & & & & & & \\
\hline \hline & & VS & S & M & L & VL \\
\hline & VL & S & M & L & Ler & Lest \\
\hline & L & Ser & S & M & L & Ler \\
\hline & M & Sest & Ser & S & M & L \\
\hline & S & Sest & Sest & Ser & S & M \\
\hline & VS & Sest & Sest & Sest & Ser & S \\
\hline
\end{tabular}

the performance of the decision-making process. The output of HFC, $\hat{f}$, is used to calculate $f_{\text {shed }}$ according to 7 .

$$
f_{\text {shed }}=f_{\text {min-shed }}+\left(f_{\text {max }- \text { shed }}-f_{\text {min-shed }}\right) \hat{f}
$$

where $f_{\text {min-shed }}$ is the minimum acceptable frequency drop for under frequency relay and $f_{\text {max-shed }}$ is the maximum set point for under frequency relay. In this paper, these parameters are equal to 42.5 and $48 \mathrm{HZ}$, respectively. Based on (2), a time variation load shedding curve is defined for under frequency relay. The flowchart of the proposed method is presented in Fig 5 .

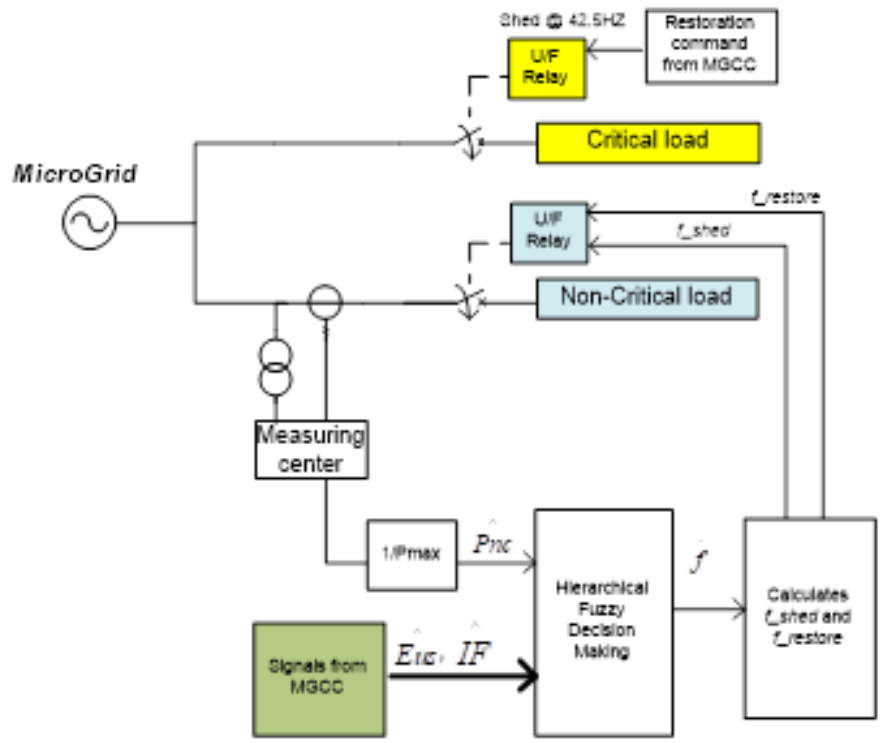

Fig. 5: Flowchart of the Problem-solving Process

\section{Simulation Results}

In this section, the proposed method is implemented on a typical MG with two generators and six load points. The nominal power of generations and loads are given in Table III. In these case studies the data is exchanged between ILCs and MGCC every 0.5 seconds; based on the past data that is in every ILCs and information that comes from MGCC, ILCs must be participated in load frequency control. In $t=2$ seconds, MG is isolated from the upstream network due to a fault. Also, in $t=3$ seconds, a mismatch occurred between active power generation and demand. The system frequency and voltage is equal to $50 \mathrm{HZ}$ and $400 \mathrm{~V}$, respectively. When IMC cannot restore the frequency, the frequency drop remains in the system and six ILCs are involved in load frequency control to restore frequency in standard boundary. In the first case study, the inputs of HFC are depicted in Table IV. According to Table IV, load shedding curves for ILCs is shown in Fig. 6. Based load shedding curves, non-critical loads no. $3,5,1$, and 2 are disconnected at $t_{1}=4.1 \mathrm{~s}, t_{2}=4.3 \mathrm{~s}$, $t 3=5.2 \mathrm{~s}, t_{4}=6.8 \mathrm{~s}$, respectively. The frequency of $\mathrm{MG}$ is depicted in Fig. 7 for different times. As it can be seen from the simulation results, after 12.5 second, the restoration happens and in $t=14$ seconds the frequency of network return to the nominal frequency $(f=50 H Z)$.

TABLE III: Nominal Power of Generation and Loads

\begin{tabular}{|c|c|c|c|c|c|c|c|}
\hline Power (kW) & 1 & 2 & 3 & 4 & 5 & 6 & 7 \\
\hline \hline Critical & 515 & 520 & 1500 & 1230 & 1020 & 1020 & 300 \\
\hline Non-Critical & 285 & 265 & 340 & 270 & 400 & 270 & 0 \\
\hline DG1 & 1100 & & & & & & \\
\hline DG2 & 6700 & & & & & & \\
\hline
\end{tabular}

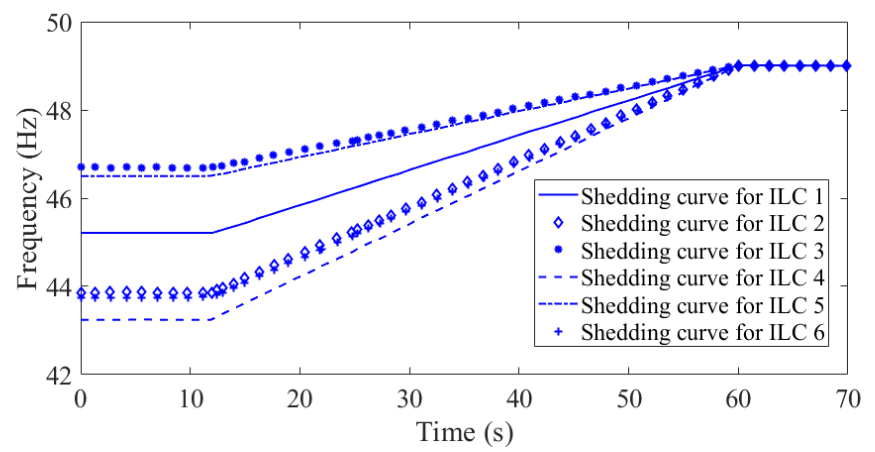

Fig. 6: Load Shedding Curves for Case I

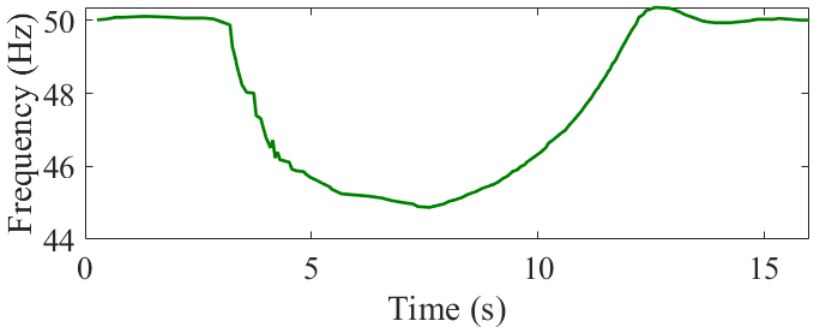

Fig. 7: Frequency of MG, Case study I

In the second case, by changing the MG conditions the value of ILC's input parameters might be change to participate in load shedding strategy. The inputs of HFC are depicted in Table V. According to Table V, load shedding curves for ILCs is shown in Fig. 8. Based load shedding curves, non-critical loads no. $6,4,5$, and 1 are disconnected at $t_{1}=4.1 \mathrm{~s}, t_{2}=4.6$ 
$\mathrm{s}, t_{3}=5.8 \mathrm{~s}, t 4=6.3 \mathrm{~s}$, respectively. The frequency of $\mathrm{MG}$ is depicted in Fig. 9 for different times.

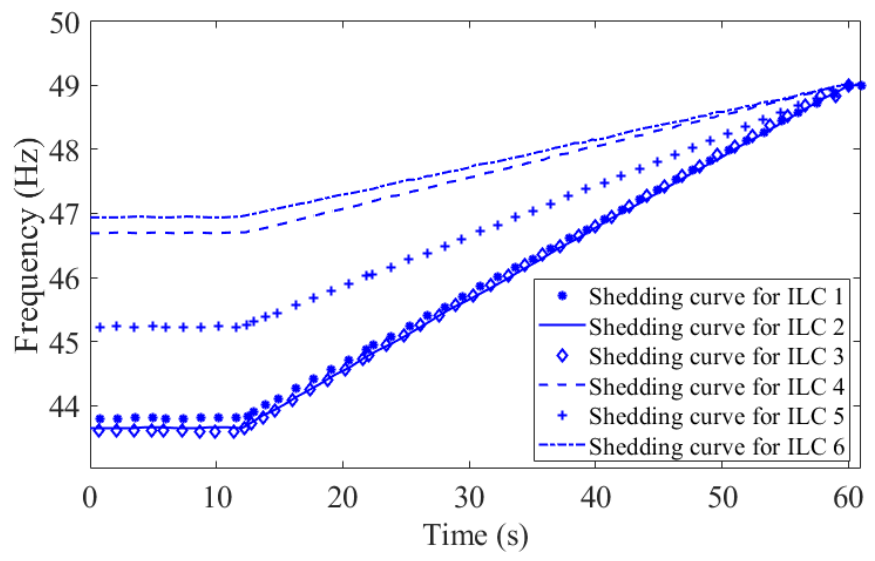

Fig. 8: Load Shedding Curves for Case II

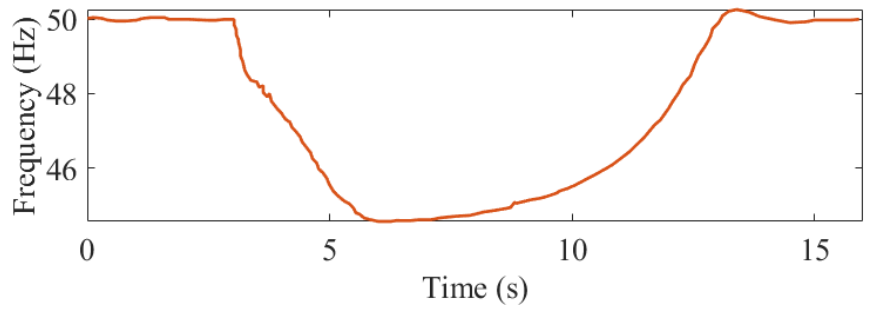

Fig. 9: Frequency of MG, case study II

In compare between case study I and II the load shedding curves of non-critical loads are changed, Fig. 6 and Fig. 8, in result, with using the proposed HFC the load shedding priority are changed and reliability indices are improved. Whereas in conventional system, the priority of load shedding may not change in a few step of frequency control and the reliability indices become worse.

TABLE IV: Inputs of ILMC's Hierarchical Fuzzy Block, Case Study I

\begin{tabular}{|c|c|c|c|}
\hline Load $i$-th & $P \hat{N} C$ & $\hat{I F}$ & $E \hat{N} S$ \\
\hline \hline 1 & 0.7 & 0.46 & 0.5 \\
\hline 2 & 0.6 & 0.81 & 0.4 \\
\hline 3 & 0.8 & 0.46 & 0.3 \\
\hline 4 & 0.4 & 0.78 & 1.0 \\
\hline 5 & 1.0 & 0.55 & 0.4 \\
\hline 6 & 0.4 & 0.57 & 0.5 \\
\hline
\end{tabular}

TABLE V: Inputs of ILMC's Hierarchical Fuzzy Block, Case Study II

\begin{tabular}{|c|c|c|c|}
\hline Load $i$-th & $P \hat{N} C$ & $\hat{I F}$ & $E \hat{N} S$ \\
\hline \hline 1 & 0.6 & 0.66 & 0.8 \\
\hline 2 & 0.6 & 0.9 & 0.6 \\
\hline 3 & 0.7 & 0.66 & 0.7 \\
\hline 4 & 0.75 & 0.3 & 0.4 \\
\hline 5 & 0.9 & 0.65 & 0.6 \\
\hline 6 & 1 & 0.3 & 0.5 \\
\hline
\end{tabular}

\section{Conclusion}

Secure and reliable operation of MGs, in both gridconnected and islanding operating modes is the most important issues during the past few years. Load frequency control is a vital problem which has a significant impact on reliable operation of MGs. Intelligent load shedding can be employed as a useful approach to control the frequency of MGs. In this paper, a novel method is suggested for optimal load shedding in MGs. In order to enhance the ability of the decentralized control system, the proposed method uses hierarchical fuzzy controller in intelligent load controllers. In this study, the reliability indices are used as the inputs of hierarchical fuzzy controller to select the optimal load shedding during frequency drop. It is notable that with much decision making variables, hierarchical fuzzy controller can effectively reduce the decision making time; consequently, MG has a convenient load controller which is enhanced by means of HFS for load frequency control.

\section{REFERENCES}

[1] E. Vaahedi, F. Rahimi, and E. Amundson, "A versatile microgrid management solution for the emerging electricity grid: Catering to the full spectrum of microgrids," IEEE Electrification Magazine, vol. 8, no. 4, pp. 91-97, 2020.

[2] M. V. Vives, H. R. Chamorro, D. Ortiz-Villalba, F. Jimenez, F. M. Gonzalez-Longatt, G. Jimenez-Estevez, J. Guerrero, A. Cadena, and V. K. Sood, "Nanogrids: Good practices and challenges in the projects in Colombia," pp. 421-446. [Online]. Available: https://digital-library.theiet.org/content/books/10.1049/pbpo160e_ch16

[3] H. R. Baghaee, M. Mirsalim, and G. B. Gharehpetian, "Performance improvement of multi-der microgrid for small- and large-signal disturbances and nonlinear loads: Novel complementary control loop and fuzzy controller in a hierarchical droop-based control scheme," IEEE Systems Journal, vol. 12, no. 1, pp. 444-451, 2018.

[4] N. N. A. Bakar, M. Y. Hassan, M. F. Sulaima, M. N. Mohd Nasir, and A. Khamis, "Microgrid and load shedding scheme during islanded mode: A review," vol. 71, pp. 161-169. [Online]. Available: https://www.sciencedirect.com/science/article/pii/S1364032116311030

[5] R. V. A. Neves, R. Q. Machado, V. A. Oliveira, X. Wang, and F. Blaabjerg, "Multitask fuzzy secondary controller for ac microgrid operating in stand-alone and grid-tied mode," IEEE Transactions on Smart Grid, vol. 10, no. 5, pp. 5640-5649, 2019.

[6] P. C. Sahu, S. Mishra, R. C. Prusty, and S. Panda, "Improved-salp swarm optimized type-II fuzzy controller in load frequency control of multi area islanded AC microgrid," vol. 16, pp. 380-392. [Online]. Available: https://www.sciencedirect.com/science/article/pii/S2352467718303369

[7] H. R. Chamorro, C. A. Ordonez, and J. F. Jimenez, "Coordinated control based petri nets for microgrids including wind farms," in 2012 IEEE Power Electronics and Machines in Wind Applications, 2012, pp. 1-6.

[8] S. Choudhury, P. Bhowmik, and P. K. Rout, "Robust dynamic fuzzybased enhanced $\mathrm{VPD} / \mathrm{FQB}$ controller for load sharing in microgrid with distributed generators," vol. 100, no. 4, pp. 2457-2472. [Online]. Available: https://doi.org/10.1007/s00202-018-0724-6

[9] R. Al Badwawi, W. R. Issa, T. K. Mallick, and M. Abusara, "Supervisory control for power management of an islanded ac microgrid using a frequency signalling-based fuzzy logic controller," IEEE Transactions on Sustainable Energy, vol. 10, no. 1, pp. 94-104, 2019.

[10] A. Ghafouri, J. Milimonfared, and G. B. Gharehpetian, "Fuzzy-adaptive frequency control of power system including microgrids, wind farms, and conventional power plants," IEEE Systems Journal, vol. 12, no. 3, pp. 2772-2781, 2018.

[11] J. L. Mirez, H. R. Chamorro, C. A. Ordonez, and R. Moreno, "Energy management of distributed resources in microgrids," in 2014 IEEE 5th Colombian Workshop on Circuits and Systems (CWCAS), 2014, pp. 1-5.

[12] A. G. Tobon, H. R. Chamorro, F. Gonzalez-Longatt, and V. K. Sood, "Reliability assessment in transmission considering intermittent energy resources," in 2019 IEEE 10th Latin American Symposium on Circuits Systems (LASCAS), 2019, pp. 193-196. 
[13] M. Khooban, T. Niknam, M. Shasadeghi, T. Dragicevic, and F. Blaabjerg, "Load frequency control in microgrids based on a stochastic noninteger controller," IEEE Transactions on Sustainable Energy, vol. 9, no. 2, pp. 853-861, 2018.

[14] D. I. Narvaez, M. G. Villalva, and E. L. Vasquez, "Fuzzy-granulation based controller for low distortion in pwm inverter with reduced load power," in 2017 Brazilian Power Electronics Conference (COBEP), 2017, pp. 1-6.

[15] M. S. Mahdavi, G. B. Gharehpetian, P. Ranjbaran, and H. Azizi, "Frequency regulation of aut microgrid using modified fuzzy pi controller for flywheel energy storage system," in 2018 9th Annual Power Electronics, Drives Systems and Technologies Conference (PEDSTC), 2018, pp. 426431.

[16] Tran Dang Khoa, L. T. Dos Santos, M. Sechilariu, and F. Locment, "Load shedding and restoration real-time optimization for dc microgrid power balancing," in 2016 IEEE International Energy Conference (ENERGYCON), 2016, pp. 1-6.

[17] Y. Hong, M. Hsiao, Y. Chang, Y. Lee, and H. Huang, "Multiscenario underfrequency load shedding in a microgrid consisting of intermittent renewables," IEEE Transactions on Power Delivery, vol. 28, no. 3, pp. 1610-1617, 2013.

[18] L. R. Srinivas and B. M. Ram, "Voltage and frequency control of distribution generation unit in an island mode microgrid using differential evolution," in 2018 Second International Conference on Intelligent Computing and Control Systems (ICICCS), 2018, pp. 772-778.

[19] R. Keypour, B. Adineh, M. H. Khooban, and F. Blaabjerg, "A new population-based optimization method for online minimization of voltage harmonics in islanded microgrids," IEEE Transactions on Circuits and Systems II: Express Briefs, vol. 67, no. 6, pp. 1084-1088, 2020.

[20] H. R. Chamorro and B. W. Toro, Control de Velocidad de Motores de Induccion y Celdas Resonantes: Aplicando Lógica Difusa.
[21] A. A. Ovalle, H. R. Chamorro, and G. Ramos, "Step-size fuzzy control to maximum power point tracking algorithms for pv microgrid arrays," in IX Latin American Robotics Symposium and IEEE Colombian Conference on Automatic Control, 2011 IEEE, 2011, pp. 1-6.

[22] A. Karaki, M. Begovic, H. Abu-Rub, S. Bayhan, and I. Poonahela, "On frequency control techniques for microgrids," in 2018 International Conference on Smart Energy Systems and Technologies (SEST), 2018, pp. 1-6.

[23] H. R. Chamorro, I. Riaño, R. Gerndt, I. Zelinka, F. Gonzalez-Longatt, and V. K. Sood, "Synthetic inertia control based on fuzzy adaptive differential evolution," International Journal of Electrical Power Energy Systems, vol. 105, pp. 803 - 813, 2019. [Online]. Available: http://www.sciencedirect.com/science/article/pii/S0142061517323785

[24] X.-Z. Wang and C.-R. Dong, "Improving generalization of fuzzy ifthen rules by maximizing fuzzy entropy," IEEE Transactions on Fuzzy Systems, vol. 17, no. 3, pp. 556-567, 2009.

[25] S. Kamthan and H. Singh, "Hierarchical fuzzy logic for multi-input multi-output systems," IEEE Access, vol. 8, pp. 206 966-206 981, 2020.

[26] G. V. S. Raju, J. Zhou, and R. A. Kisner, "Hierarchical fuzzy control," vol. 54, no. 5, pp. 1201-1216. [Online]. Available: https://doi.org/10.1080/00207179108934205

[27] M. Hajiakbari Fini and M. E. Hamedani Golshan, "Frequency control using loads and generators capacity in power systems with a high penetration of renewables," vol. 166, pp. 43-51. [Online]. Available: https://www.sciencedirect.com/science/article/pii/S0378779618303031

[28] D. Wang, X. Zeng, and J. A. Keane, "Intermediate variable normalization for gradient descent learning for hierarchical fuzzy system," IEEE Transactions on Fuzzy Systems, vol. 17, no. 2, pp. 468-476, 2009.

[29] H. R. Chamorro and M. A. Ríos, "Pss fuzzy controllers with new defuzzification strategies for a multimachine system," in 2010 IEEE ANDESCON, 2010, pp. 1-6. 